\title{
Increased Lateral Femoral Condyle Ratio Measured by MRI Is Associated with Higher Risk of Noncontact Anterior Cruciate Ligament Injury
}

\author{
Miao He ( $\sim$ smallhem@163.com ) \\ Chongqing Emergency Medical Center(Chongqing University Central Hospital) \\ Jie Li \\ Chongqing Emergency Medical Center(Chongqing University Central Hospital)
}

\section{Research Article}

Keywords: Anterior cruciate ligament, Knee, Lateral femoral condyle ratio, Femur

Posted Date: November 23rd, 2021

DOI: https://doi.org/10.21203/rs.3.rs-1087807/v1

License: (c) (1) This work is licensed under a Creative Commons Attribution 4.0 International License.

Read Full License

Version of Record: A version of this preprint was published at BMC Musculoskeletal Disorders on March 1st, 2022. See the published version at https://doi.org/10.1186/s12891-022-05134-x. 


\section{Abstract \\ Background}

Studies have shown a significant association between the radiographically measured lateral femoral condyle ratio (LFCR) and anterior cruciate ligament (ACL) injury. However, it is unclear whether the the LFCR measured by magnetic resonance imaging (MRI) is associated with risk of noncontact ACL injury.

\section{Objective}

1 To investigate the effect of the LFCR on the risk of noncontact ACL injury by MRI. 2 To investigate the association of the LFCR measured by MRI with multiple bone morphological risk factors and evaluate the most sensitive risk predictors of noncontact ACL injury.

\section{Methods}

A total of 116 patients, including 58 subjects with noncontact ACL injury and 58 age-matched and sexmatched controls with only meniscus injury, were included in this retrospective case-control study. The LFCR, lateral tibial slope (LTS), lateral tibial height (LTH), medial tibial slope (MTS), and medial tibial depth (MTD) were measured on MRI. The differences in each index between the two groups were compared, and risk factors were screened by single-factor logistic regression analysis. Indicators with $\mathrm{P}$ values $<0.1$ were included in the logistic regression equation. The critical values and areas under the curve (AUCs) of independent risk factors were determined by receiver operating characteristic (ROC) curve analysis. Finally, the diagnostic performance of each risk factor was evaluated by the Z-test.

\section{Results}

A total of 116 patients who met the inclusion criteria were included in the final analysis (58 cases in the noncontact ACL injury group and 58 cases in the control group). Patients with noncontact ACL injury had a higher femoral LFCR $(63.5 \pm 2.7 \%)$ than patients with simple meniscus tear. Among all the risk factors for ACL injury, the AUC for the LFCR was the largest, at 0.81 (95\% Cl, 0.73-0.88), and when the critical value was $61.35 \%$, the sensitivity and specificity for the diagnosis of ACL injury were $79 \%$ and $67 \%$, respectively. Combined with the LTH $(>2.35 \mathrm{~mm})$, the diagnostic performance was improved. The AUC was $0.85(95 \% \mathrm{Cl}, 0.78-0.92)$, the sensitivity was 0.83 , and the specificity was 0.76

\section{Conclusion}

This study shows that an increased LFCR is related to an increased risk of noncontact ACL injury by MRI. The LFCR and LTH are the most sensitive risk factors for noncontact ACL injury and may help clinicians 
identify individuals prone to ACL injury, allowing prevention and intervention measures to be applied.

\section{Background}

The rate of noncontact anterior cruciate ligament (ACL) injury has increased substantially in athletes [1]. An increasing number of scholars have begun to pay close attention to targeted prevention and treatment, and early assessment and identification of ACL injury risk factors is necessary to reduce the incidence of this kind of injury and to avoid long-term damage caused by knee joint instability, resulting in cartilage wear and early knee joint osteoarthritis [2].

With the deepening of the understanding of anatomical factors affecting ACL injury, the study of bone morphometry of the tibia and femur has received extensive attention. Medial tibial compartment depression [3] and posterior tibial plateau tilt [4, 5] are associated with increased ACL load and risk of injury. The asymmetry of the medial and lateral compartments with medial tibial concavity and lateral convexity, as well as asymmetry of the distal femoral condylar shape, contribute to the pivoting mechanism [6, 7]. Thus, axial movement occurs, further causing ACL injury [8].

The bony morphology of the lateral femoral compartment is currently of considerable interest because it may play an important role in the phenomenon of pivot shift and knee kinematics $[9,10,11]$. Studies have suggested that the LFCR measured by X-ray plays a role in noncontact ACL injury of the knee joint [12]. Whether the LFCR measured by magnetic resonance imaging (MRI) is a risk factor for noncontact $A C L$ injury is unclear. The main purpose of this study was to evaluate the shape of the lateral femoral compartment by MRI and investigate its impact on the risk of noncontact ACL injury. The secondary objective was to evaluate the most sensitive risk factors for noncontact ACL injury by combining measurements with multiple bone morphological risk factors. Our hypothesis was that the LFCR, as measured by MRI, was associated with the risk of noncontact ACL injury.

\section{Materials And Methods}

\section{Study design}

A total of 612 patients who underwent arthroscopic surgery for ACL injury, or simple meniscus tear or who underwent MRI for anterior knee pain at The Affiliated Central Hospital of Chongqing University from 2017 to 2021 were retrospectively analysed. Patients were divided into the following groups: (1) the noncontact ACL injury group and (2) the control group; the control group included those with simple meniscus tear without ligament injury and no signs of patellofemoral dysplasia. Patients in the control group were matched by sex and age with those in the treatment group and then assessed for the eligibility criteria listed in Fig. 1. All patients underwent MRI, and scans were read by senior radiologists and surgeons to determine the presence of a ruptured ACL or torn meniscus.

\section{Instruments and equipment}


A 1.5T MRI scanner (1.5T, GE Signa, GE Healthcare, USA) was used to measure the knee joint parameters of the study subjects at Central Hospital affiliated with Chongqing University. Sagittal, coronal, and axial sequences of MRI T1 and T2 stages were included, with a thickness of $3.0 \mathrm{~mm}$ each.

\section{MRI data measurement method}

Referring to the method of Pfeifer for X-ray measurement of the LFCR [6], the LFCR was measured by MRI. Figure A shows the sagittal T1 centre of a knee on MRI, in which the insertion of the posterior cruciate ligament, intercondylar spine, and anterior and posterior cortices are convex. To determine the long axis of the distal femur, two circles were drawn at the centre of the femur axis. The more distant circle was placed at the nearest end of the tackle. A line passing through the centre of the two circles was considered to be the long axis of the distal femoral axis. The MRI T1 sagittal centre of the lateral femoral condyle was then selected to replicate the long axis of the distal femur, and the axis of the femoral condyle was determined by drawing a line between the last point and the most anterior point of the lateral condyle. The distance from the intersection of these two lines to the last point of the condyle was divided by the total length of the condyle and multiplied by $100 \%$. This ratio was defined as the lateral femoral condyle ratio (Figure 2).

BABA1012In this study, bone morphological indicators including the medial tibial slope (MTS) [13], lateral tibial slope (LTS) [13], medial tibial depth (MTD) [14], and lateral tibial height (LTH) [15] were measured by MRI using the methods of previous studies (Figure 3).

\section{ABCDE}

All measurements were made by 2 blind reviewers (HeM, LiJ) to ensure interobserver reliability for the entire cohort. To assess intraobserver reliability, all measurements were repeated 3 weeks later with a reader $(\mathrm{HeM})$.

\section{Statistical analysis}

IBM SPSS 20.0 (IBM Corp. New York, USA) and MedCalc 12.7 (MedCalc Software bvba, Ostend, Belgium) were used for the statistical analyses. The significance level was set at 0.05. Quantitative data are presented as the mean \pm standard deviation. The receiver operating characteristic (ROC) curve sample size estimation yielded a required sample size of 56 patients (alpha, 0.05; power, 0.8). The Kolmogorov-

Smirnov test was used to assess whether the variables followed a normal distribution. The Wilcoxon rank sum test was used to compare the morphological parameters (LFCR, LTS, LTH, MTD) in the noncontact $A C L$ rupture group and the control group, and the paired $T$ test was used to compare the morphological parameters (MTS) with a normal distribution. There were statistically significant differences in the LFCR, LTS, LTH, MTS and MTD. All indicators were included in the single-factor analysis, and indicators with a P value $<0.1$ were included in binary logistic regression analysis. The LFCR and LTH were screened as independent risk factors for noncontact ACL injury, and the prediction probability of the combined LFCR and LTH was calculated. The critical value and area under the curve (AUC) were calculated by ROC curve 
analysis to evaluate the clinical diagnostic efficacy of the three risk factors (LFCR, LTH, and LFCR+LTH). The AUC values for each risk factor were compared with the Z-test using MedCalc 12.7 software.

\section{Ethical approval}

Informed consent was obtained from all participants or, if participants are under 16, from a parent and/or legal guardian, and the study was approved by the Committee of Central Hospital affiliated with Chongqing University.

\section{Results}

A total of 116 patients were included in the final analysis (58 in the noncontact ACL injury group and 58 in the control group). Each group consisted of 20 females and 38 males, with an average age of $31.05 \pm 9.28$ years in the noncontact ACL injury group and $30.76 \pm 9.38$ years in the control group. The intraobserver reliability for the LFCR was 0.86 ( $95 \% \mathrm{Cl}, 0.80-0.90)$, and the interobserver reliability for the LFCR was 0.85 ( $95 \% \mathrm{Cl}, 0.79-0.90)$. (Table 1)

\section{TABLE 1 Inter- and Intraobserver Reliability Among All MRI Measurements Performed ${ }^{a}$}

\begin{tabular}{|lll|}
\hline & Interobserver Reliability & Intraobserver Reliability \\
\hline LFCR $\% \%$ Q & $0.85(0.79-0.90)$ & $0.86(0.80-0.90)$ \\
\hline LTS & $0.85(0.78-0.90)$ & $0.83(0.77-0.88)$ \\
\hline LTH & $0.84(0.77-0.89)$ & $0.87(0.82-0.91)$ \\
\hline MTS & $0.90(0.85-0.93)$ & $0.89(0.84-0.92)$ \\
\hline MTD & $0.82(0.72-0.88)$ & $0.84(0.78-0.89)$ \\
\hline
\end{tabular}

a Values are presented as intraclass correlation coefficients (95\% $\mathrm{Cl})$. MRI, magnetic resonance imaging. LFCR, lateral femoral condyle index Ratio. LTS, lateral tibial slope. LTH, lateral tibial height. MTS, medial tibial slope. MTD, medial tibial depth.

The LFCR in the noncontact ACL injury group (63.5 $\pm 2.7 \%)$ was significantly higher than that in the control group $(60.3 \pm 2.5 \%)(P<0.01)$. The LTS, LTH, MTS and MTD in the noncontact ACL injury group were higher than those in the control group (Wilcoxon rank sum tests were used for comparisons of LFCR, LTS, LTH and MTD, and paired T tests were used for comparison of MTS) (Table 2).

Sex differences in the LFCR between the noncontact ACL injury group and the control group were not statistically significant. The mean LFCR was $63.95 \pm 2.61 \%$ in males and $62.75 \pm 2.78 \%$ in females in the noncontact ACL injury group. In the control group, the mean LFCR was $60.27 \pm 2.12 \%$ in males and $60.35 \pm 3.07 \%$ in females. 
TABLE 2 Bone morphological indexes among groups ${ }^{a}$

\begin{tabular}{|llll|}
\hline & Non-contact ACL injury $(n=58)$ & Control Group $(n=58)$ & P Value \\
\hline LFCR $^{\mathrm{b}}(\%)$ & $63.54 \pm 2.71$ & $60.29 \pm 2.46$ & $<0.01$ \\
\hline LTS $^{\mathrm{b}}$ & $8.13 \pm 3.85$ & $6.40 \pm 3.77$ & 0.03 \\
\hline LTH $^{\mathrm{b}}$ & $2.81 \pm 0.76$ & $2.33 \pm 0.58$ & $<0.01$ \\
\hline MTS $^{\mathrm{c}}$ & $7.36 \pm 3.45$ & $6.01 \pm 3.01$ & 0.046 \\
MTD $^{\mathrm{b}}$ & $2.83 \pm 0.71$ & $2.59 \pm 0.60$ & 0.04 \\
\hline
\end{tabular}

avalues are expressed as the mean \pm standard deviation. LFCR, lateral femoral condyle index ratio. LTS, lateral tibial slope. LTH, lateral tibial height. MTS, medial tibial slope. MTD, medial tibial depth.

${ }^{b} p$ values refer to the Wilcoxon rank sum test.

${ }^{c} p$ values refer to the paired $T$ test.

TABLE 3 Univariate and multivariate analyses of all MRI measurements ${ }^{a}$

\begin{tabular}{|lllll|}
\hline & Univariate analysis & \multicolumn{3}{l|}{ Multivariate analysis } \\
\cline { 3 - 5 } & P value & P value & OR & OR:95\% Cl \\
\hline LFCR $(\%)$ & $<0.01$ & $<0.01$ & 1.68 & $1.35-2.10$ \\
\hline LTS & 0.02 & 0.49 & 1.05 & $0.91-1.22$ \\
\hline LTH & $<0.01$ & $<0.01$ & 2.65 & $1.28-5.46$ \\
\hline MTS & 0.03 & 0.18 & 1.16 & $0.96-1.39$ \\
\hline MTD & 0.05 & 0.66 & 1.19 & $0.55-2.61$ \\
\hline
\end{tabular}

${ }^{a}$ MRI, magnetic resonance imaging; LFCR, lateral femoral condyle index ratio; LTS, lateral tibial slope; LTH, lateral tibial height; MTS, medial tibial slope; MTD, medial tibial depth; OR, odds ratio.

TABLE 4 Diagnostic Performance Among the Three MRI Measurements ${ }^{a}$ 


\begin{tabular}{|llllllll|}
\hline & Sensitivity & Specificity & $\begin{array}{l}\text { Youden } \\
\text { index }\end{array}$ & $\begin{array}{l}\text { Cut-off } \\
\text { value }\end{array}$ & AUC & AUC:95\%Cl & $\begin{array}{l}\text { P } \\
\text { value }^{\mathrm{b}}\end{array}$ \\
\hline LFCR (\%) & 0.79 & 0.67 & 0.47 & 61.35 & 0.81 & $0.73-0.88$ & - \\
\hline LTH & 0.74 & 0.57 & 0.31 & 2.35 & 0.69 & $0.60-0.79$ & 0.06 \\
\hline LFCR+LTH & 0.83 & 0.76 & 0.59 & 0.46 & 0.85 & $0.78-0.92$ & 0.06 \\
\hline
\end{tabular}

\section{aMRI, magnetic resonance imaging; LFCR, lateral femoral condyle ratio; LTH, lateral tibial height; AUC, area under the curve.}

\section{${ }^{b} \mathrm{P}$ value of each AUC tested against the AUC for the LFCR; binomial Z-test.}

Single-factor logistic regression analysis was performed to screen potential risk factors, and the LFCR $(P<0.01)$, LTS $(P=0.02)$, LTH $(P<0.01)$, MTS $(P=0.03)$, and MTD $(P=0.05)$ were included in the logistic regression equation. Independent risk factors associated with an increased risk of noncontact ACL injury were identified as the LFCR (OR, 1.68; 95\% $\mathrm{Cl}, 1.35-2.10)(\mathrm{P}<0.01)$ and LTH (OR, 2.65; 95\% Cl, 1.28-5.46) $(\mathrm{P}<0.01)$ (Table 3).

Among all the independent risk factors, the LFCl had the highest AUC $(0.81 ; 95 \% \mathrm{Cl}, 0.73-0.88)$, and the sensitivity, specificity and cut-off values for predicting noncontact ACL injury were $79 \%, 67 \%$, and $61.35 \%$, respectively (Youden index $=0.47$ ) (Table 4 and Figure 4 ).

According to the logistic regression analysis, the AUC for the LFCR+LTH was 0.85 ( $95 \% \mathrm{Cl}, 0.78-0.92)$, with a sensitivity of 0.83 and specificity of 0.76 for predicting noncontact ACL injury (Table 3 ). However, the AUC for the LFCR+LTH was not significantly different from that of the LFCR alone $(P=0.06)$ (Table 4).

\section{Discussion}

The current study found that an increased LFCR, as measured by MRI, was associated with an increased risk of noncontact ACL injury. The LFCR threshold for predicting noncontact ACL rupture was $61.35 \%$, with a sensitivity of $79 \%$ and specificity of $67 \%$, which confirmed the hypothesis of the study. Combining the two most predictive factors ( $\mathrm{LFCR}>61.35 \%$ and $\mathrm{LTH}>2.35 \mathrm{~mm}$ ) improved the predictive diagnostic performance for noncontact ACL fracture. The AUC was 0.85 , sensitivity was 0.83 , and specificity was 0.76 . These parameters can help clinicians identify patients at risk for ACL injury.

Many studies have shown that the shape of the lateral femoral condyle has an important influence on the rotational stability of the knee [15,9]. Pfeiffer reported that the mechanism of the influence of lateral femoral condyle shape on knee rotation stability was an increase in the depth of the lateral femoral posterior condyle, which may affect the movement of the femur in relation to the tibia and lead to changes in gait and load mechanics [8]. The increase in the depth of the lateral posterior condyle of the femur alters the shape of the lateral condyle of the femur to be more elliptical and nonequidistant, which may lead to an increase in ligament relaxation when the knee joint is nearly fully extended [8]. It 
may also lead to a reduction in the contact area between the femur and tibia, thereby increasing rotational relaxation of the knee [8]. The results of this study are consistent with the aforementioned results.

In this study, we found that the combination of the two most predictive factors (LFCR>61.75\%, LTH>2.35 $\mathrm{mm}$ ) was of better value in diagnosing and predicting noncontact ACL injuries than either factor alone. This confirms previous findings that bone morphology of both the femoral and tibial lateral ventricles contributes to axial displacement of the knee. That is, increased depth of the lateral posterior condyle of the femur and height of the lateral tibial plateau lead to increased rotation $[9,16]$. These differences in bone morphology affect the biomechanics of the knee, causing greater traction of the ACL and increasing the risk of injury [17].

In this study, there was no significant difference in the LFCR between women and men. In previous studies, sex differences in the morphology of the distal femur were reported. Some studies found that the anatomical morphology of femurs in females was inconsistent with that of femurs in males [11,18], while other studies found that there was no significant difference in the morphology of distal femurs between males and females [10].

Previous studies have used different measurement methods to describe the bony morphology of the lateral femoral condyle and further found that bony morphology changes in the lateral femoral condyle could increase the possibility of ACL injury. Pfeiffer quantitatively measured the osseous morphology of the lateral condyle of the femur by the ratio of the lateral condyle on X-ray [11]. The method was simple and relatively accurate. The exclusion of a large number of patients because of malrotated radiographs could introduce bias. The study by Voleti et al. also demonstrated that radiographs underestimate posterior condylar depth measurements when compared with MRI [19]. The use of MRI would have allowed the authors to accurately quantify the posterior condylar depths of the lateral femoral condyles, assess their influence on the risk of ACL injury and reduce measurement imprecision and patient exclusion because of malrotated radiographs. Hodel et al. used the LFCl to quantitatively measure the skeletal morphology of the lateral femoral condyle on MRI [10]. In other words, the flexion circle and extension circle were drawn before and after the lateral femoral condyle, respectively, and the ratio of the radius of the two circles was considered the lateral femoral condyle index [10]. However, Li et al. believed that the two circles had great uncertainty and randomness, so this measurement method may have large errors [20].

This study has some strengths but also some limitations. The main advantages of this study are that the LFCR was measured by MRI, and this method is simple to apply. Reducing the exclusion of large numbers of patients due to poor $\mathrm{X}$-ray rotation may introduce bias. Considering several risk factors, a diagnostic threshold was proposed. The limitations of this study are as follows: Compared with X-ray, MRI is expensive and thus not conducive to large-scale screening and prevention. Knee computed tomography (CT) can be used to calculate the LFCR in future research. 


\section{Conclusion}

In this study, an elevated LFCR was associated with noncontact ACL injury by MRI, and the LFCR and LTH were the most predictive risk factors for noncontact ACL injury. This may help clinicians identify individuals prone to ACL injury, allowing targeted prevention measures and interventions.

\section{Abbreviations}

LFCR: lateral femoral condyle ratio; ACL: anterior cruciate ligament; MRI: magnetic resonance imaging; LTS: lateral tibial slope; LTH: lateral tibial height; MTS: medial tibial slope; MTD: medial tibial depth; AUCs: areas under the curve; ROC: receiver operating characteristic.

\section{Declarations}

\section{Acknowledgements}

Not Applicable.

\section{Authors' contributions}

All authors contributed to the study conception and design. Material preparation, data collection and analysis were performed by HM and LJ. The first draft of the manuscript was written by HM, LJ made meaningful correction to the structure of the article and guided the statistical methods and data processing, and all authors commented on previous versions of the manuscript. HM participated in the design of the study and proofread the manuscript as the corresponding author. All authors have read and approved the manuscript.

\section{Funding}

Funding information are not available.

\section{Availability of data and materials}

All raw data and materials during the study are available from the first author by request. (He Miao, smallhem@163.com).

\section{Ethics approval and consent to participate}

This retrospective study involving human participants was in accordance with the ethical standards of the institutional and national research committee and with the 1964 Helsinki Declaration and its later amendments or comparable ethical standards. The study was approved by the Committee of Central Hospital affiliated with Chongqing University. Informed consent was obtained from all participants or, if participants are under 16 , from a parent and/or legal guardian. 


\section{Consent for publication}

Not Applicable.

\section{Competing interests}

The authors declare that they have no competing interests.

\section{Author details}

Department of Orthopaedic Surgery, Chongqing Emergency Medical Center (Chongqing University Central Hospital), Chongqing 400010, China

\section{References}

1. Gianotti SM, Marshall SW, Hume PA, et al. Incidence of anterior cruciate ligament injury and other knee ligament injuries: a national population-based study. 2010, 12(6): 622-7.

2. Swart E, Redler L, Fabricant PD, et al. Prevention and screening programs for anterior cruciate ligament injuries in young athletes: a cost-effectiveness analysis. 2014, 96(9): 705-11.

3. Hashemi J, Chandrashekar N, Mansouri $\mathrm{H}$, et al. Shallow medial tibial plateau and steep medial and lateral tibial slopes: new risk factors for anterior cruciate ligament injuries. Am J Sports Med. 2017; 38:54-62.

4. Dare DM, Fabricant PD, McCarthy MM, et al. Increased lateral tibial slope is a risk factor for paediatric anterior cruciate ligament injury: an MRI-based case-control study of 152 patients[J]. Am J Sports Med, 2015, 43(7): 1632-1639.

5. Elmansori A, Lording T, Dumas R, et al. Proximal tibial bony and meniscal slopes are higher in ACL injured subjects than controls: a comparative MRI study[J]. Knee Surg Sports Traumatol Arthrosc, 2017, 25(5):1598-1605.

6. Pfeiffer T, Burnham J, Herbst E, Shafizadeh S, Musahl V. A Tomahawk shape of the femur predicts greater rotatory knee laxity in patients with anterior cruciate ligament ruptures. Orthop J Sports Med. 2018;6(4) (suppl 2):2325967118S0003.

7. Siebold R, Axe J, Irrgang JJ, Li K, Tashman S, Fu FH. A computerized analysis of femoral condyle radii in intact and contralateral ACL reconstructed knees using 3D CT. Knee Surg Sports Traumatol Arthrosc. 2009; 18:26-31.

8. Pfeiffer TR, Burnham JM, Kanakamedala AC, Hughes JD, Zlotnicki J, Popchak A, Debski RE, Musahl V. Distal femur morphology affects rotatory knee instability in patients with anterior cruciate ligament ruptures. Knee Surg Sports Traumatol Arthrosc. 2019 May;27(5):1514-1519.

9. Fernandes MS, Pereira R, Andrade R, Vasta S, Pereira H, Pinheiro JP, et al. Is the femoral lateral condyle's bone morphology the trochlea of the ACL? Knee Surg Sports Traumatol Arthrosc. 2017;25(1):207-214. 
10. Hodel S, Kabelitz M, Tondelli T, Vlachopoulos L, Sutter R, Fucentese SF. Introducing the lateral femoral condyle index as a risk factor for anterior cruciate ligament injury. Am J Sports Med. 2019;47(10):2420-2426.

11. Pfeiffer TR, Burnham JM, Hughes JD, Kanakamedala AC, Herbst E, Popchak A, et al. An increased lateral femoral condyle ratio is a risk factor for anterior cruciate ligament injury. J Bone Joint Surg Am. 2018;100(10):857-864.

12. Li K, Zheng X, Li J, Seeley RA, Marot V, Murgier J, Liang X, Huang W, Cavaignac E. Increased lateral femoral condyle ratio is associated with greater risk of ALC injury in non-contact anterior cruciate ligament injury. Knee Surg Sports Traumatol Arthrosc. 2021 Sep;29(9):3077-3084.

13. Hudek R, Schmutz S, Regenfelder E, Fuchs B.Novel Measurement Technique of the Tibial Slope on Conventional MRI[J].Clin Orthop Relat Res, 2009, 467:2066-2072.

14. Hashemi J, Chandrashekar N, Mansouri H, et al. Shallow medial tibial plateau and steep medial and lateral tibial slopes: new risk factors for anterior cruciate ligament injuries. Am J Sports Med. 2017; 38:54-62.

15. Kujala UM, Nelimarkka O, Koskinen SK. Relationship between the pivot shift and the configuration of the lateral tibial plateau. Arch Orthop Trauma Surg. 1992; 111:228-229.

16. Wahl CJ, Westermann RW, Blaisdell GY, et al. An association of lateral knee sagittal anatomic factors with non-contact ACL injury: Sex or geometry? Journal of Bone and Joint Surgery - Series A, 2012, 94(3): 217-26.

17. Stijak L, Herzog RF, Schai P. Is there an influence of the tibial slope of the lateral condyle on the ACL lesion? A case- control study. Knee Surgery, Sports Traumatology, Arthroscopy, 2008, 16(2): 112-7.

18. Conley S, Rosenberg A, Crowninshield R. The female knee: anatomic variations. J Am Acad Orthop Surg. 2007;15(suppl 1): S31-S36

19. Voleti PB, Stephenson JW, Lotke PA, Lee GC. Plain radiographs underestimate the asymmetry of the posterior condylar offset of the knee compared with MRI. Clin Orthop Relat Res. 2014 Jan;472(1):155-61.

20. Li R, Liu Y, Fang Z, Zhang J. Introducing the Lateral Femoral Condyle Index as a Risk Factor for Anterior Cruciate Ligament Injury: Letter to the Editor. Am J Sports Med. 2020 Jun; 48(7):NP42.

\section{Figures}




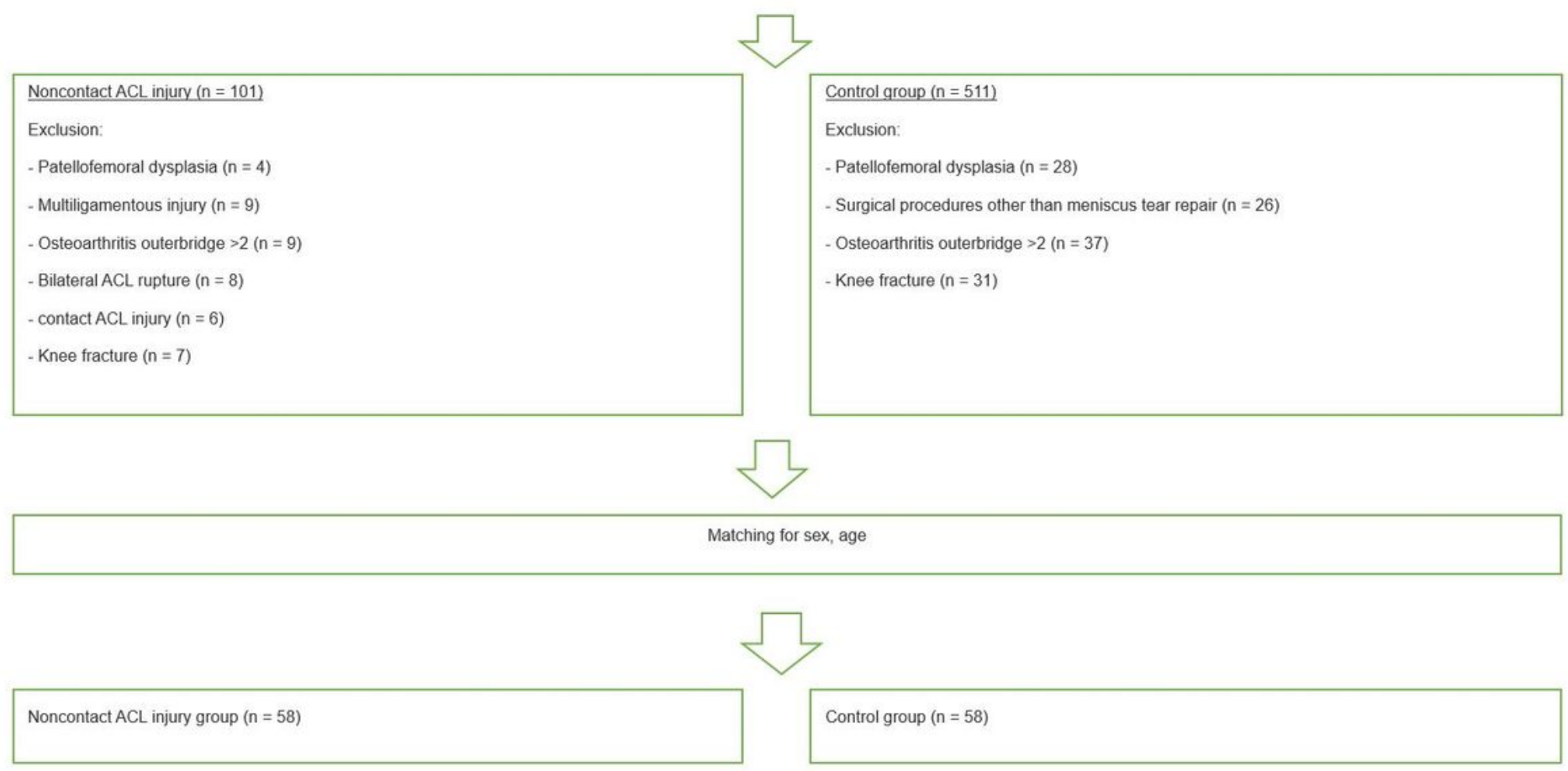

\section{Figure 1}

Flowchart and eligibility. ACL, anterior crucial ligament; MRI, magnetic resonance imaging.

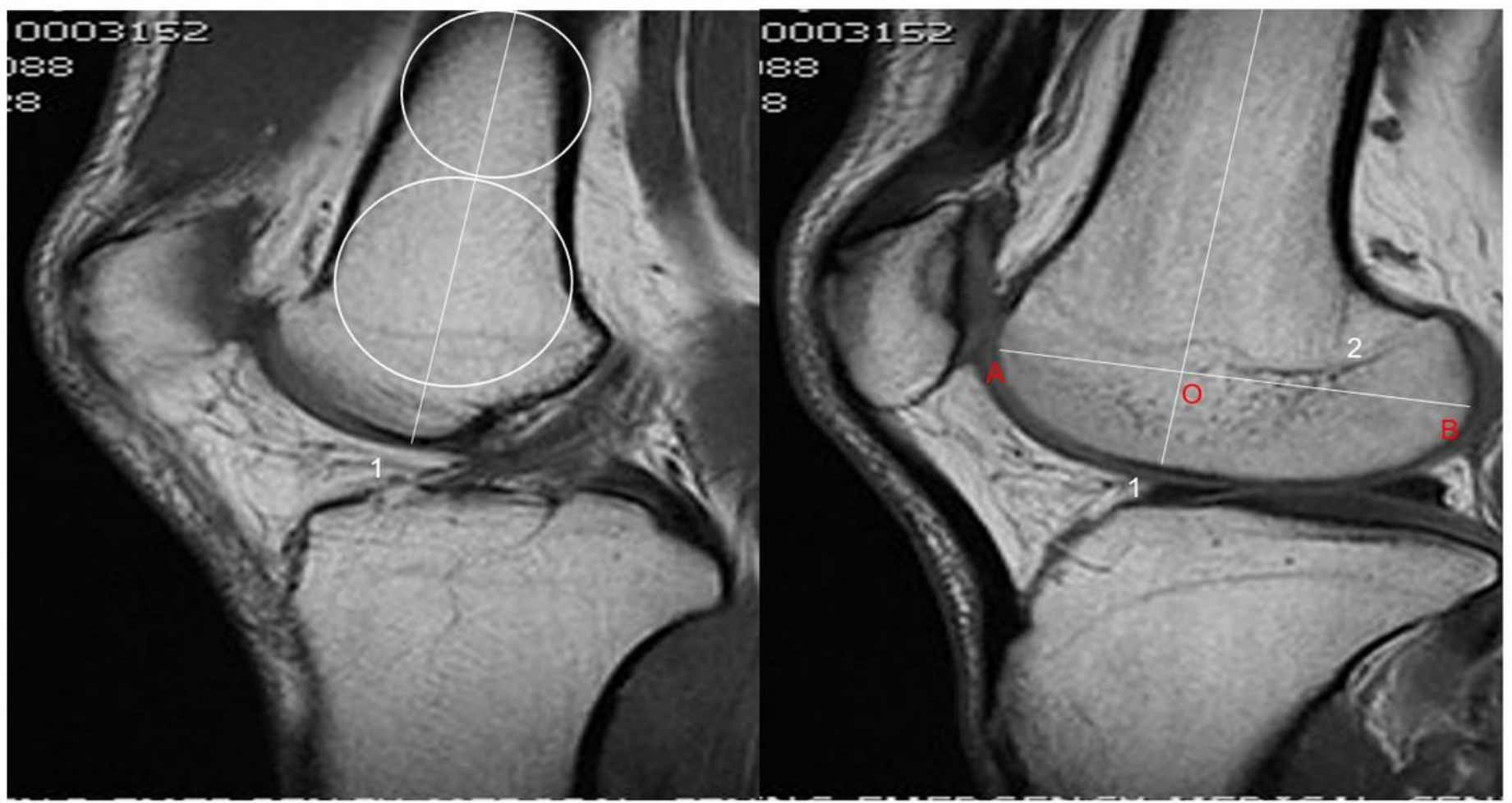

Figure 2 
Measurement of the LFCR: (A) In the sagittal T1 centre of the knee MRI, 2 circles were drawn in the centre of the femoral axis to determine the long axis of the distal femur. The more distant circle was placed at the nearest end of the tackle. The line passing through the centre of the two circles was considered to be the long axis of the distal shaft of the femur (segment 1). (B) In the sagittal T1 MRI centre of the lateral condyle of the femur, the long axis of the distal femur (segment 1 ) was replicated. The axis of the femoral condyle was then determined by drawing a line (line segment 2 ) between the last point of the lateral condyle (point B) and the most anterior point (point A). The distance from the intersection of the two lines (point 0 ) to the last point of the condyle was divided by the total length of the condyle, i.e., $\mathrm{OB} / \mathrm{AB}$, and multiplied by 100 . This ratio was defined as the lateral femoral condyle ratio.

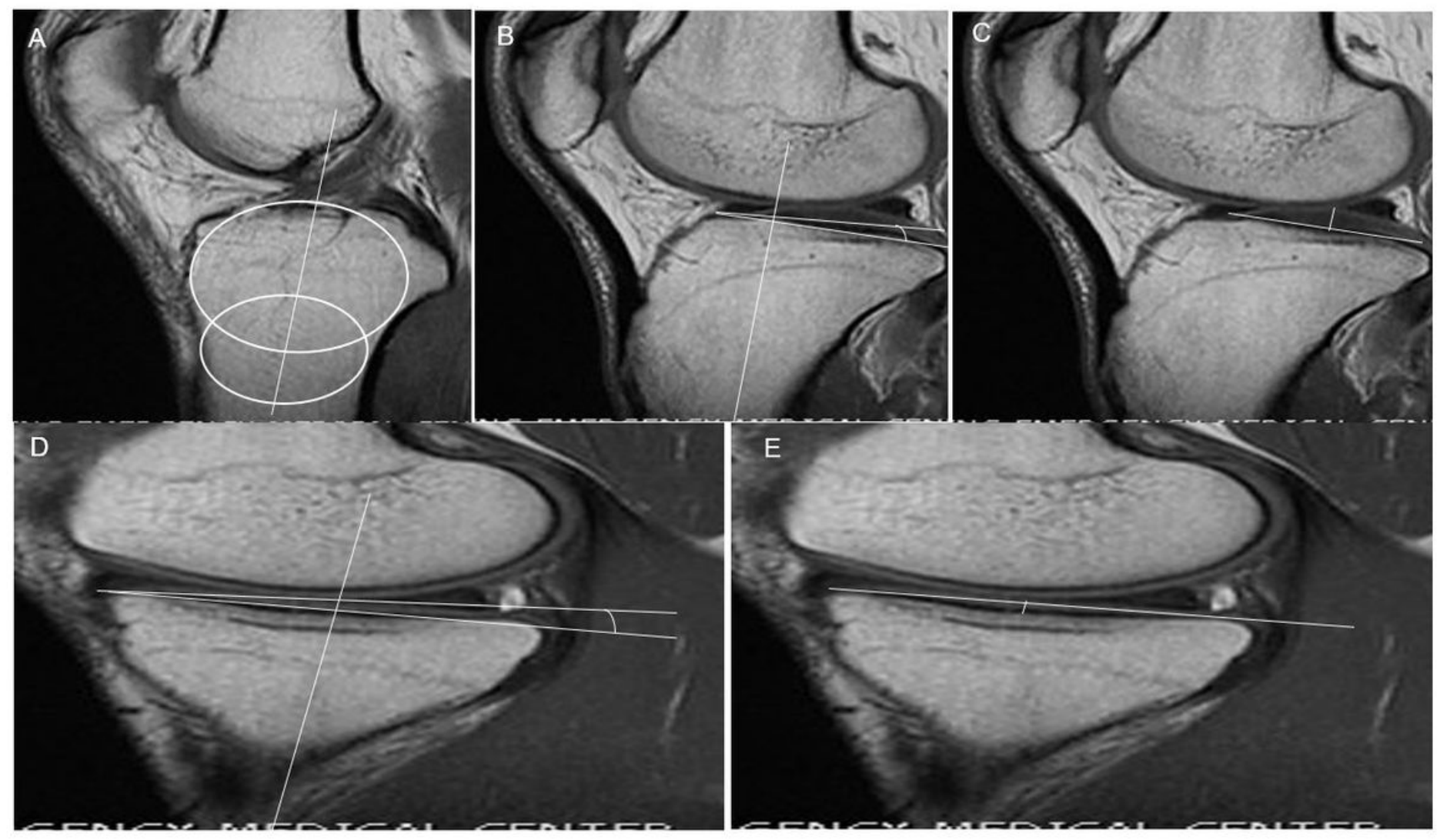

\section{Figure 3}

As described previously, various indicators of bone morphology were measured by MRI: (A) midsagittal tibial reference, (B) lateral tibial slope (LTS) [13], (C) lateral tibial height (LTH) [15], (D) medial tibial slope (MTS) [13], and (E) medial tibial depth (MTD) [14]. 
ROC Curve

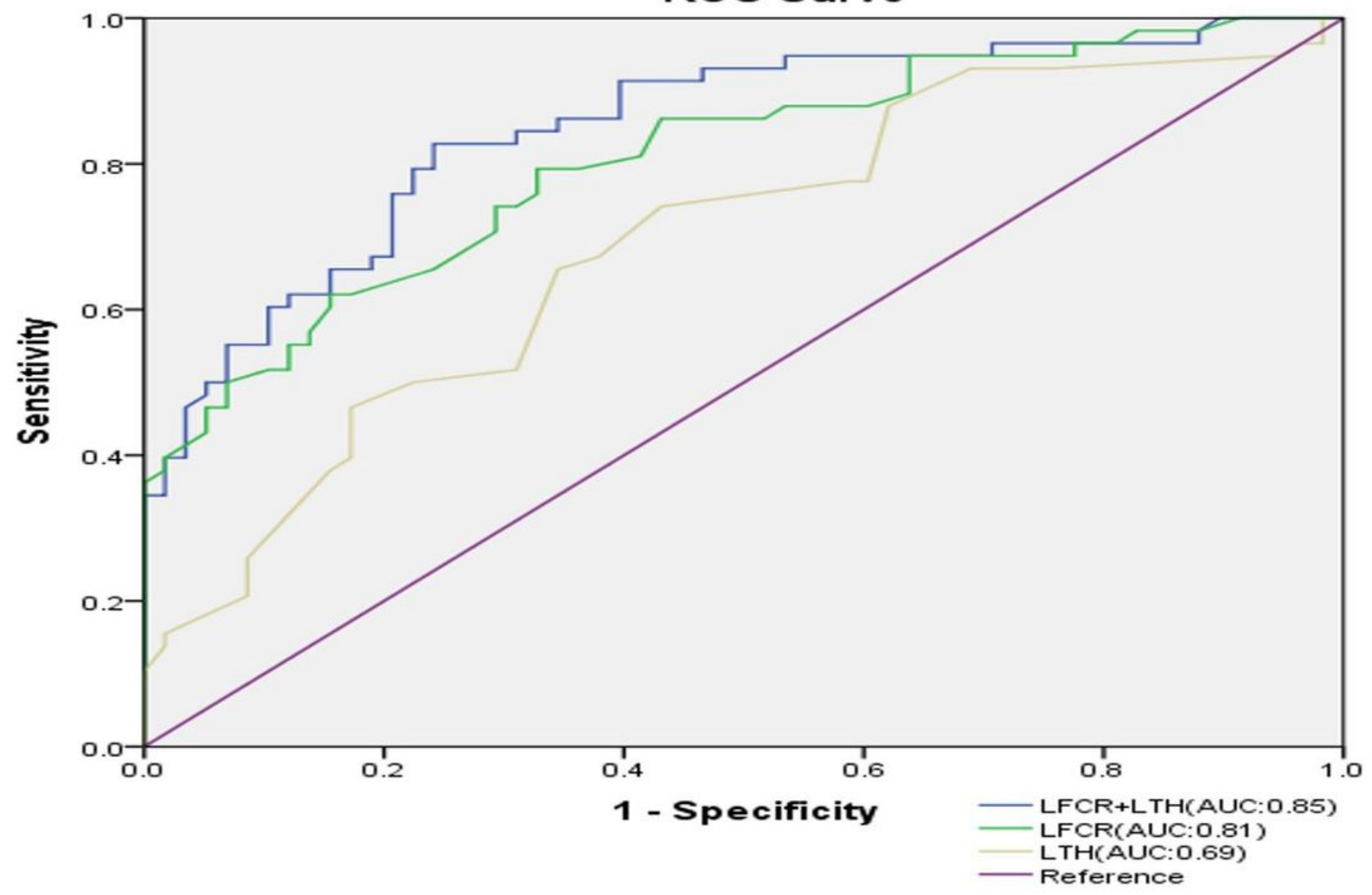

Figure 4

Receiver operating characteristic curves for the LFCR, LTH, and LFCR+LTH. Reference line: AUC $=0.5$. AUC, area under the curve; $\mathrm{LFCl}$, lateral femoral condyle ratio; LTH, lateral tibial height. 